\title{
Microwave Tomographic approach for masonry investigation: some real results
}

\author{
M. Bavusi ${ }^{1}$, R. Di Napoli ${ }^{2}$, and F. Soldovieri ${ }^{2}$ \\ ${ }^{1}$ Istituto per le Metodologie di Analisi Ambientale, Consiglio Nazionale delle Ricerche, C. da S. Loja Zona Industriale, \\ 85050 Tito Scalo (PZ), Italy \\ ${ }^{2}$ Istituto per il Rilevamento Elettromagnetico dell'Ambiente, Consiglio Nazionale delle Ricerche, Via Diocleziano 328, \\ 80124 Napoli, Italy
}

Received: 10 February 2010 - Revised: 1 March 2010 - Accepted: 11 March 2010 - Published: 27 April 2010

\begin{abstract}
Ground Penetrating Radar (GPR) is an electromagnetic technique very appreciated by the community of the archaeologist and cultural heritage end-users community thanks to its appealing features in terms of non invasivity and rapidity of measurement and diagnostics. However, GPR data requires a high operator expertise in the data processing and interpretation. In the archaeological investigation, this drawback can be mitigated by the availability of a priori information about the archaeological scenario. On the other hand, in the case of the historical heritage, when the knowledge of constructive modalities and material of the structure may be completely missed, it is necessary to undertake other strategies of processing and interpretation.

One of these can be provided by the use of novel inversion algorithms such as the Microwave Tomography (MT) which allows to reduce the subjectivity and the time consuming during the data processing. In this paper the MT was applied on raw data collected at two historical buildings of Chania (Crete, Greece). The first edifice is the Venizelo's House affected by visible fractures in its walls made up of cemented irregular stones. The second one is the headquarters of Prefecture of Chania showing some fractures along the floors. For these raw data, microwave tomography provided well detailed images which allowed to infer the fracture geometry and their extension in the host medium. This suggests microwave tomography can be a reliable complete processing tool requiring only the definition of the background scenario in terms of the dielectric permittivity and the conductivity of the host medium.
\end{abstract}

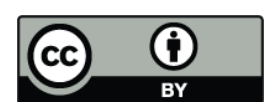

Correspondence to: F. Soldovieri (soldovieri.f@irea.cnr.it)

\section{Introduction}

Ground Penetrating Radar (GPR) is one of the most feasible and friendly instrumentation to detect buried remains and perform diagnostics of historical structures (buildings, temples, masonries) with the aim of detecting embedded objects: therefore, its employ is well assessed in archaeological prospecting when the aim is to detect and locate buried remains thus providing a fruitful support to excavations planning (Daniels 2004; Conyers and Goodman, 1997; Piro et al., 2003).

On the other side, its use is assessed also in the case of cultural heritage diagnostics as for example in masonry diagnostics (defects, voids, constructive typology; etc.) or foundations investigation (Binda et al., 1998).

The increasing use of the GPR technique is mainly due to the possibility to perform measurements over large areas in a very fast way thanks to a portable instrumentation (Daniels, 2004). In particular, GPR is based on the phenomenon of the electromagnetic scattering made possible by exploiting an antenna system (made up of transmitting and receiving antennas). Its simple operating principle is based on a transmitting antenna emitting an electromagnetic wave that impinges on the buried target; the interaction incident wavetarget arises a field back-scattered by the target that is after collected by the receiving antenna.

Usually, GPR is exploited in a configuration, where the receiving and transmitting antennas are separated by a small fixed offset and are moved very close to or in contact with the ground-interface (B-scan). In this way, a time domain trace is collected for each antenna system's position and after all the traces are joined and processed in order to visualize the B-scan radargram. The resulting radargram is in

Published by Copernicus Publications on behalf of the European Geosciences Union. 
general "low interpretable" and entails a high operator's expertise and the availability of a priori information about the scene is required to achieve a comprehensible "interpretation and visualization" of the scene.

On the other hand, the archaeologists community is mainly interested in visualization of the investigated scene that has to be easily interpretable over a large scale and also able to give details (high resolution diagnostics) of the investigated site when necessary. This difficulty of the interpretation is exacerbated when no a priori information is available as, for example, arises in the case of historical heritages when the knowledge of the constructive modalities and materials of the structure might be completely missed.

A possible bridge between the GPR technicians and the GPR end-users community is now possible thanks to the development and exploitation of new data processing based on the inverse scattering problem (Catapano et al., 2006; Soldovieri and Orlando, 2009). In particular, the ones based on microwave tomography (Leone and Soldovieri, 2003; Soldovieri et al., 2005) are gaining increasing interest. These approaches are based on more accurate formulation of the electromagnetic scattering phenomenon model and robust reconstruction procedures when compared to the ones usually adopted in the classic radaristic approach (Soldovieri et al., 2007; Bavusi et al., 2008) Therefore, the exploitation of the microwave tomographic approach allows to gives an answer to the issue of gaining accurate and reliable "images" of the investigated structure in order to detect, localize and possibly determine the extent and the geometrical features of the embedded objects.

In this paper, we present a microwave tomography based approach able to tackle the applicative necessities of the archaeological prospecting/cultural heritage diagnostics, which consist in the possibility of investigating large investigation scenes and reducing at the minimum level of the subjectivity in the data processing with an improvement of the fastness of the investigation.

Therefore, the paper is organized as follows. Section 2 is devoted to present the microwave tomographic approach and mathematical detail about the inversion procedure are briefly recalled. Section 3 is devoted at describing the sites and giving the details about the GPR survey. Section 4 presents the outcomes of the microwave tomography based approach and for sake of comparison also the results achieved by the classical radaristic approach are presented. Finally conclusions follow.

\section{The microwave tomographic approach}

The microwave tomographic here presented tackles a linear inverse scattering problem thanks to the adoption of the Born Approximation (Chew, 1995; Leone and Soldovieri, 2003; Crocco and Soldovieri, 2003). The approach is accurately described in several papers, see for instance (Leone and Sol-

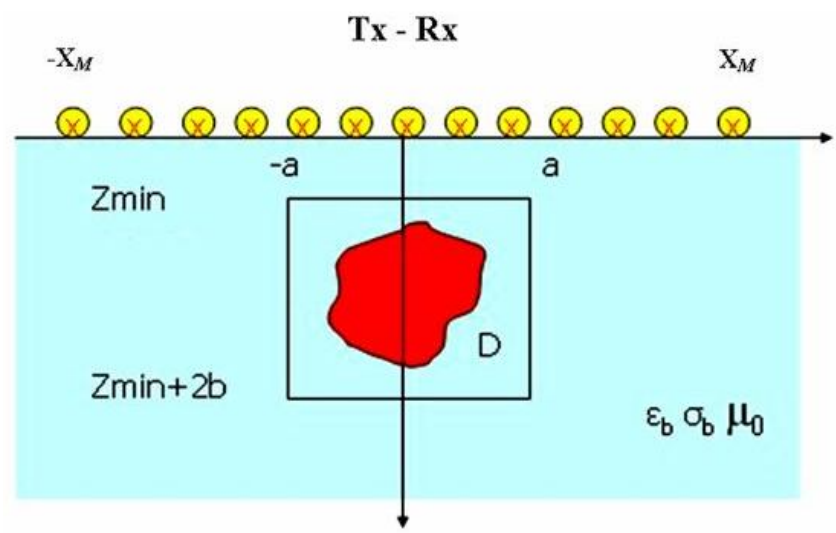

Fig. 1. Geometry of the two-dimensional inverse scattering problem.

dovieri, 2003; Persico et al., 2005) and here we only briefly sketch its main features. The approach is formulated with respect to the two-dimensional geometry that is depicted in Fig. 1. The inhomogeneous background scenario is modeled as two homogeneous half-spaces separated by a planar interface at $z=0$. The upper half-space is the air (dielectric permittivity $\varepsilon_{0}$ ) where the antenna system moves, whereas the lower one representing for example the soil (for archaeology prospecting) or a masonry (as an example for the cultural heritage) that is assumed to have a relative dielectric permittivity $\varepsilon_{b}$ and an electrical conductivity $\sigma_{b}$.

The incident field source is simply modeled as a timeharmonic (time dependence $\exp (\mathrm{j} 2 \pi \mathrm{ft})$ ) filamentary $\mathrm{y}$ directed electric current, invariant along the $y$-axis that represents the axis orthogonal to the investigation plane. The data are collected under a multi-frequency multi-monostatic configuration characterized by the fact that the locations of transmitting and receiving antennas coincide, the field is collected under a work frequency band $\left(f_{\min }, f_{\max }\right)$.

The targets to be imaged are assumed to be invariant along the $y$-axis (2-D geometry) and their cross-section is enclosed in the rectangular investigation domain D (see Fig. 1).

In particular, the targets are represented in terms of the relative dielectric permittivity and conductivity anomalies with respect to the properties of the unaltered medium (in this case the host soil with parameters $\varepsilon_{b}$ and $\sigma_{b}$ ).

Accordingly, the inverse problem is recast in terms of the unknown contrast function which indeed embeds such "anomalies" given as target relative dielectric permittivity $\varepsilon_{r}\left(x^{\prime}, z^{\prime}\right)$ and conductivity $\sigma\left(x^{\prime}, z^{\prime}\right)$ with respect to the background and is defined as:

$\chi\left(x^{\prime}, z^{\prime}\right)=\frac{\varepsilon_{e q}\left(x^{\prime}, z^{\prime}\right)-\varepsilon_{\text {eqb }}}{\varepsilon_{\text {eqb }}}$

$\varepsilon_{\mathrm{eqb}}=\varepsilon_{0} \varepsilon_{b}-j \frac{\sigma_{b}}{2 \pi f}$ and $\varepsilon_{e q}\left(x^{\prime}, z^{\prime}\right)=\varepsilon_{0} \varepsilon_{r}\left(x^{\prime}, z^{\prime}\right)-j \frac{\sigma\left(x^{\prime}, z^{\prime}\right)}{2 \pi f}$ being the equivalent complex dielectric permittivity of the 
targets and of the soil, respectively. Notice that the unknown contrast function $\chi(x, z)=0$ when $(x, z)$ does not belong to the support of the target.

The inverse scattering problem can be simplified by assuming an approximated model based on the Born approximation (Leone and Soldovieri, 2003; Persico et al., 2005; Crocco and Soldovieri, 2003); in this case, the relationship in the frequency domain between the unknown contrast function and the scattered field data is provided through a linear integral equation:

$E_{S}\left(x_{s}, \omega\right)=k_{s}^{2} \int_{D} G_{e}\left(x_{s}, \omega, \boldsymbol{r}^{\prime}\right) E_{\mathrm{inc}}\left(x_{s}, \omega, \boldsymbol{r}^{\prime}\right) \chi\left(\boldsymbol{r}^{\prime}\right) d \boldsymbol{r}^{\prime}$

where $\omega=2 \pi f$ is the angular frequency; $k_{s}$ is the wavenumber in the soil; $E_{s}\left(x_{s}, \omega\right)$ is the electric scattered field along the air-soil interface collected at the abscissa xs and frequency $f$ and represents the datum of our problem.

By definition, the scattered field $E_{s}$ is given by the "difference" between the total field and the unperturbed field. The total field is the sum of two contributions: the field reflected by the soil and the field backscattered by the buried objects. The unperturbed field coincides with the field reflected by the soil when the objects are absent, and therefore accounts for the reflection/transmission at the air/soil interface. Under the measurement configuration at hand, the scattered field is collected by moving the antenna system over a rectilinear observation domain at the air-soil interface with $x_{s}$ ranging from $-\mathrm{X}_{M}$ to $\mathrm{X}_{M}$ (see Fig. 1).

$G_{e}\left(x_{s}, \omega, \boldsymbol{r}^{\prime}\right)$ is the Green's function of the problem, while $E_{\text {inc }}$ is the unperturbed or incident field in the investigation domain D, i.e, the field in absence of the targets. Both these quantities are known or can be evaluated after defining the sources, the reference scenario and the measurement configuration. For a deeper understanding of their meaning and for their expression the reader is addressed to (Leone and Soldovieri, 2003; Persico et al., 2005).

The problem is then cast as the inversion of the linear integral Eq. (2) and the numerical implementation of the solution algorithm requires the discretization of Eq. (2). This task is pursued by resorting to the method of moments (MoM) (Harrington, 1961). In particular, the linear integral relationship in (2) is discretized into a linear algebraic system, where the unknowns are the expansion coefficients of the contrast function along the chosen functional basis and point-matching is adopted in the data space (Crocco and Soldovieri, 2003; Persico et al., 2005).

The inversion of the resulting matrix $\mathbf{L}$ is performed by a scheme, based on the Truncated Singular Value Decomposition (TSVD) (Bertero and Boccacci, 1998), which is able to provide robust solutions with respect to the uncertainties and the noise on data. This "regularised solution" is given by:

$\tilde{\chi}(\boldsymbol{r})=\sum_{n=0}^{N} \frac{1}{\sigma_{n}}\left\langle E_{s}, v_{n}\right\rangle u_{n}$ where $\left\{\sigma_{n}, u_{n}, v_{n}\right\}_{n=0}^{K}$ is the singular system of the matrix $\mathbf{L}$, $\langle\cdot, \cdot\rangle$ denotes the scalar product in the data space, $K$ denotes the number of measurements and $N \leq K$.

In particular, $\sigma_{n}$ are the singular values, while $v_{n}$ and $u_{n}$ are the singular vectors in the data and unknown spaces, respectively (Bertero and Boccacci, 1998). The choice of the $N$ index is performed so to ensure the trade-off between the contrasting needs of accuracy and resolution (that pushes to increase such an index) and the stability of the solution (that pushes to limit the increase of the $N$ index).

\section{Description of the sites and data acquisition}

Here, we present the application of the microwave tomographic approach to realistic data collected by GPR surveys performed at two important historical buildings of the Chania City (Crete, Greece), such as the Venizelos's House and Prefecture of Chania. The overall aim of the survey was to investigate the fracture affecting floors and walls of the above said buildings (Fig. 2).

In general, fractures represent the effects of the dynamic behavior of the building under a seismic action and can, in their turn, affect the seismic behaviour of the building. The investigation of the fractures can provide information about the building structural defects and so represents a support for the restoration/reinforcement planning project.

From the electromagnetic point of view, the fracture can be detected only when a valuable contrast of its electromagnetic and geometric properties arises a not negligible (compared to the noise and data uncertainties) scattered field; this situations is favourable affected when the fracture is filled by air or a material different from the host medium. Moreover, a fracture is generally orthogonal to the plan of the survey surface and therefore a good horizontal resolution is needed to localize it (Tsoflias et al., 2004). In this framework, Ground Penetrating Radar (GPR) measurements provides the adequate spatial resolution when a work frequency ranging from 1000 to $2000 \mathrm{MHz}$ is exploited together an advanced data processing approach able to achieve all the allowable spatial resolution (Grandjean and Gourry, 1996).

For the survey at hand, high frequency GPR survey lines have been acquired at the Prefecture of Chania building and the Venizelos' house.

The first site is the house where the revolutionary patriot Eleftherios Venizelos (1864-1836), prime minister and the "father of the modern Greece" lived. This edifice is now a museum and is of enormous historical and symbolic significance for the Greece; unfortunately, in the last year this edifice was affected by visible fractures at the masonries made up of cemented irregular stones.

The second site is the Headquarter of the Prefecture of Chania, an ancient edifice with a quadrangular shape in plan view consisting of four office buildings $60 \mathrm{~m}$ long with two or 

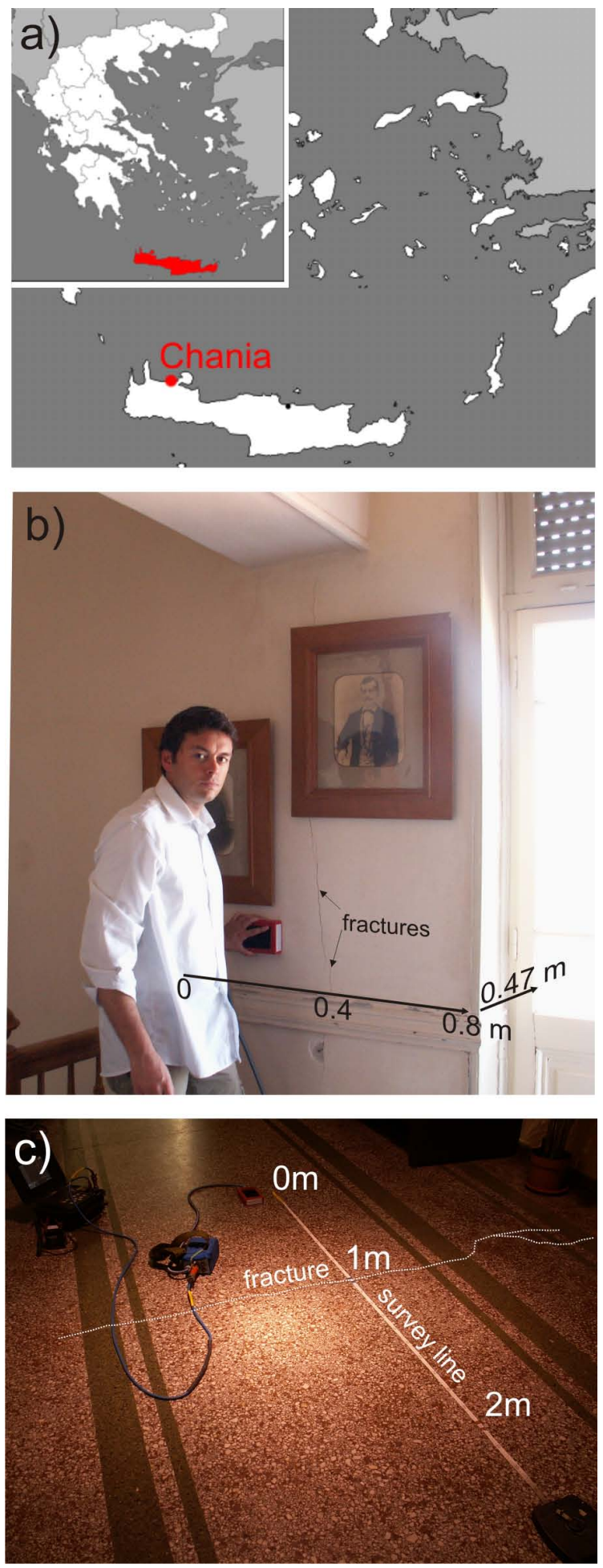

Fig. 2. (a) Crete island map; (b) survey line on a wall of Venizelos house; (c) survey carried out on a floor of Prefecture of Chania building. three floors linked at right angle so to form an internal courtyard. This edifice exhibits some fractures along the floors.

Here, we present the results for a survey line, $0.80 \mathrm{~m}$ long carried out on a wall of Venizelos' house and a fracture $2 \mathrm{~m}$ long in a floor of Prefecture of Chania building (Fig. 2b and c). In both cases, the surveys are performed so that the visible fractures fall at the centre of the survey line. This location corresponds to $0.40 \mathrm{~m}$ for Venizelos' house survey and $1.0 \mathrm{~m}$ for the Prefecture. The radargrams have been acquired by using the GSSI system with the SIR2000 control unit equipped with a $1.5 \mathrm{GHz}$ (nominal peak frequency) antenna. A scan rate of 32 scans/second, 512 samples/scans and a 16 bit sampling was selected for the survey.

\section{Data processing and results}

This section is devoted to present the results of the microwave tomographic approach and compare them with the ones achieved by the classical processing.

Classical time domain processing routine consisting of three stages: pre-processing, filtering and migration.

Pre-processing consists of a geometrical "formatting" of the raw data performed through trace removal, trace normalization, zero time correction and time gating. Basically, a pre-processed data is a geometrically corrected raw data. An example of a pre-processed radargram of Venizelos' house is shown in Fig. 3a.

Filtering stage includes several 1-D and 2-D filters (frequency filtering, subtracting average and fk filtering, etc.) with the aim of improving the signal to noise ratio.

Finally, migration has been performed by assuming a know value of the electromagnetic velocity inside the host medium.

For Venizelos' house, an electromagnetic velocity of $0.125 \mathrm{~m} / \mathrm{ns}$ has been estimated; this value was obtained by dividing the double thickness of the masonry $(0.47 \mathrm{~m})$ for the two-travel-time (3.75 ns).

Figure $3 \mathrm{~b}$ shows pre-processed filtered and migrated radargram acquired in the Venizelos' house in terms of normalized absolute value of the amplitude. Several vertical reflections can be seen and are probably related to the inner structure of the wall but no fracture evidence has been detected near the fracture zone.

The last migration step has been replaced by microwave tomography described in Sect. 2 and the result is presented in Fig. 3c. Microwave tomography assumes the investigation domain with a minimum depth of about $0.05 \mathrm{~m}$ so to cut-off the reflections of the interface air/masonry. The inversion procedure highlights several anomalous zones related to the inhomogeneous structure of the wall. Anyway, the method is able to image, in the fracture zone, two vertical structures well correspond to two branches of the fracture outcropping in this zone. Moreover, two vertical structures seem not interest all the thickness of the wall. 

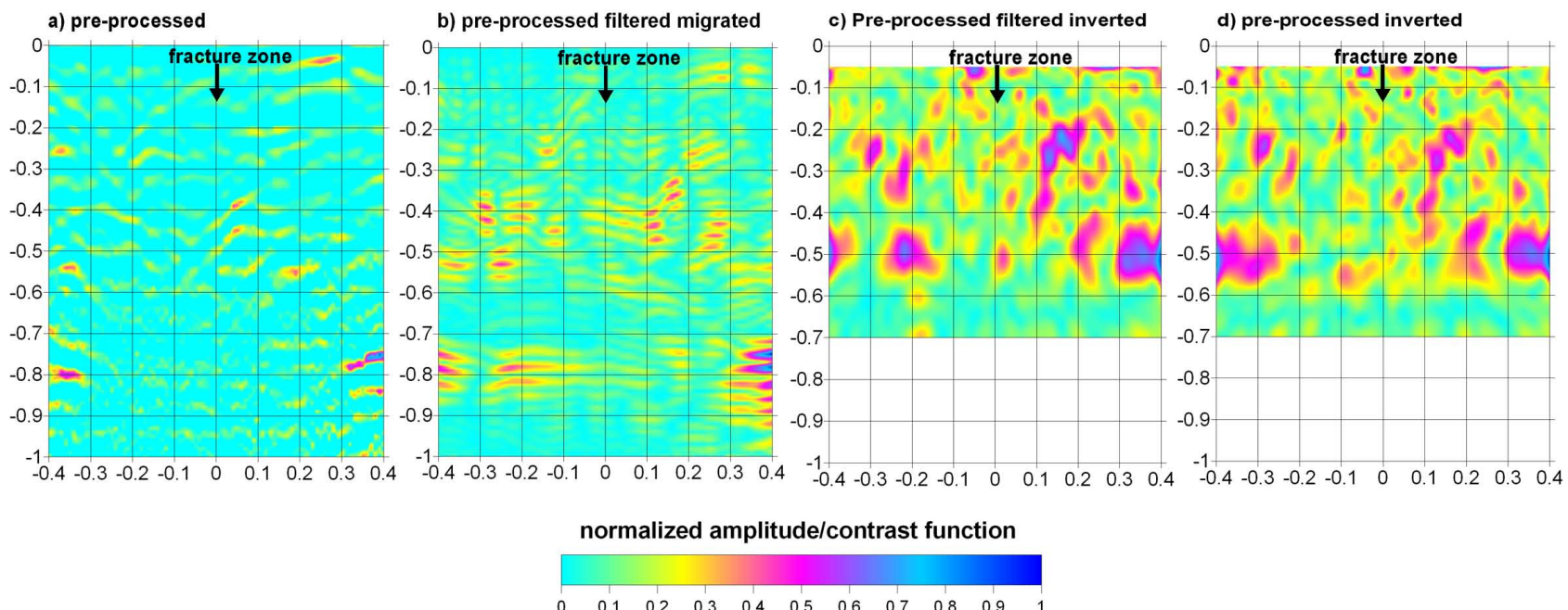

Fig. 3. Radargram carried out in correspondence of a wall of the Venizelos house. (a) preprocessed (raw) data; (b) time domain full processed data; (c) microwave tomography carried out on the pre-processed filtered data; (d) microwave tomography carried out on the pre-processed data in (a).

a) classical time domain processing results

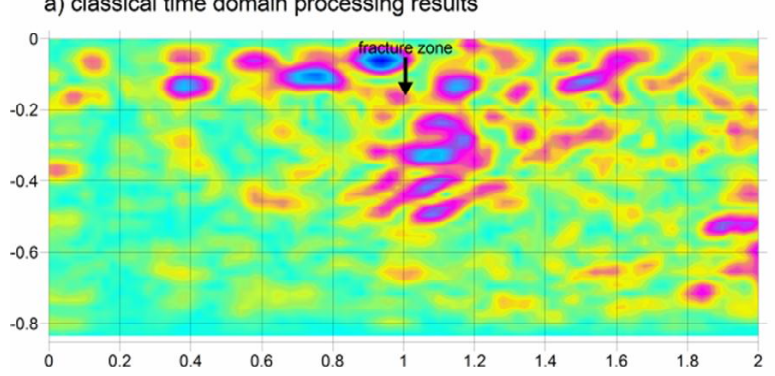

0.9

\section{b) microwave tomography results}

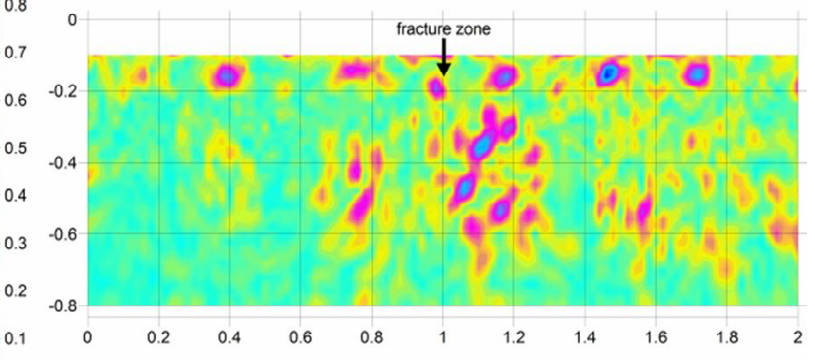

Fig. 4. Radargram carried out on a floor of the Prefecture of Chania building. (a) full classical time domain processing; (b) microwave tomography results.

Figure $3 \mathrm{~d}$ depicts the reconstruction results when the second and third stage of the classical processing are not performed. This result is very similar to the one of Fig. 3c indicating that the microwave tomography can be even applied on not-filtered data set.

The second test case is concerned with the Prefecture of Chania building and here data set has been processed starting directly by the pre-processed data (Fig. 4).

In this case the improvement of spatial resolution and of detection capability is more remarkable than the previous case due to the more homogeneous nature of the investigated medium that makes the adopted Born model close to the "reality".

The results comparison show how, starting from a preprocessed filtered data, microwave tomography provides more detailed images and improves the horizontal resolu- tion compared to the result achieved by the migration approach. Moreover, the microwave tomography performed on a merely pre-processed data show a result of the same quality when compared to the one achieved by the pre-processed and filtered data. This suggests that the microwave tomography can be a reliable complete and automatic processing tool requiring only the definition of the reference scenario, i.e., the dielectric permittivity and the conductivity of the host medium (soil, floor, wall).

\section{Conclusions}

The paper has dealt with the joint application of the Ground Penetrating Radar (GPR) and microwave tomography in the framework of the cultural heritage diagnostics. In particular, the presented cases are concerned with surveys at the 
Venizelos's House and Prefecture of Chania that are two important historical buildings of the Chania City (Crete, Greece). The aim of these investigations was to gain information about the fracture phenomenon affecting floors and walls.

The performances of the MT approach are compared with the ones of a standard time-domain processing scheme. In both the cases, the MT provided more detailed and interpretable images which allowed to infer information about the geometry and the extent of the buried part of the fractures. This suggests that microwave tomography can be a reliable complete processing tool able to reduce the requirement of operator expertise.

Acknowledgements. This work was financed by the international project "Advanced techniques for Seismic RISK Reduction in Mediterranean Archipelago Regions" (SE RISK).

Edited by: L. Eppelbaum, N. Masini, and F. Soldovieri

Reviewed by: two anonymous referees

\section{References}

Bertero, M. and Boccacci, P.: Introduction to Inverse Problems in Imaging, Philadelphia, PA, USA, IOP Publishing, 1998.

Bavusi, M., Piscitelli, S., Soldovieri, F., Crocco, L., Piscitelli, S., Prisco, G., and Vallianatos, F.: Exploitation of a microwave tomographic approach for GPR data processing collected at historical buildings of Chania (Crete, Greece), Proc. of 1st International Workshop on Advances in Remote Sensing for Archaeology and Cultural Heritage Management, Rome, Italy, 30 September-4 October, 2008.

Binda, L., Lenzi, G., and Saisi, A: NDE of masonry structures: use of radar tests for characterization of stone masonries, NDT\&E Int., 31(6), 411-419, 1998.

Chew, C. W.: Waves and Fields in inhomogeneous media, Piscataway, NJ: IEEE Press, 1995.

Catapano, I., Crocco, L., Persico, R., Pieraccini, M. and Soldovieri, F.: Linear and Nonlinear Microwave Tomography Approaches for Subsurface Prospecting: Validation on Real Data, IEEE Antenn. Wireless Propag. Lett., 5, 49-53, 2006.
Conyers, L. B. and Goodman, D.: Ground Penetrating Radar: An Introduction for Archaeologists, AltaMira Press, Walnut Creek, London and New Delhi, 1997.

Crocco, L. and Soldovieri, F.: GPR prospecting in a layered medium via microwave tomography, Ann. Geophys.-Italy., 46, 559-572, 2003.

Daniels, D.: Ground Penetrating Radar, 2nd edn. IEE Press, London, UK, 2004.

Grandjean, C. and Gourry, J. C.: GPR data processing for 3-D fracture mapping in a marble quarry (Thassos, Greece), J. Appl. Geophys., 36, 19-30, 1996.

Harrington, R. F.: Time-Harmonic Electromagnetic Fields, Mc Graw Hill, 1961.

Leone, G. and Soldovieri, F.: Analysis of the distorted Born approximation for subsurface reconstruction: truncation and uncertainties effect, IEEE T. Geosci. Remote, 41, 66-74, 2003.

Persico, R., Bernini, R., and Soldovieri, F.: The role of the measurement configuration in inverse scattering from buried objects under the Born approximation, IEEE Trans. Antennas and Propagation, 53(6), 1875-1887, 2005.

Piro, S., Goodman, D., and Nishimura, Y.: The study and characterization of Emperor Traiano's villa (Altopiani di Arcinazzo, Roma) using high-resolution integrated geophysical surveys, Archaeol. Prospect., 10, 1-25, 2003.

Soldovieri, F., Persico, R., and Leone, G.: Frequency diversity in a linear inversion algorithm for GPR prospecting, Subsurface Sensing Technologies and Applications Journal, Special Issue GPR2004, 6, 25-42, 2005.

Soldovieri, F., Hugenschmidt, J., Persico, R. and Leone, G.: A linear inverse scattering algorithm for realistic GPR applications, Near Surf. Geophys., 5, 29-42, 2007.

Soldovieri, F. and Orlando, L.: Novel tomographic based approach and processing strategies for multi-frequency antennas GPR measurements using multi-frequency antennas, J. Cult. Herit., 10, e83-e92, 2009.

Tsoflias, G. P., Van Gestelz, J. P., Blankenship, D. D. and Sen, M.: Vertical fracture detection by exploiting the polarization properties of ground-penetrating radar signals, Geophysics, 69, 803810, 2004. 